\title{
Complejidad social al final del período formativo tardío. La ceniza de la última erupción del volcán Pululahua (2400 A.P.) como marcador temporal ${ }^{*}$
}

Silvia Figueroa ${ }^{\star *}$

RESUMEN

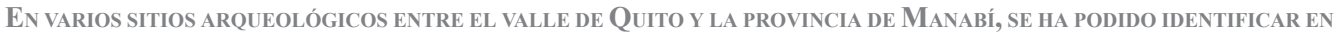
Su estratigrafía la(s) tefra(s) del último ciclo eruptivo del volcán Pululahua (2500 a 2400 años AP). EN LAS ZONAS AFECTADAS POR ESTE FENÓMENO NATURAL, ESTA CAPA DE CENIZA ES UN REFERENTE CRONOESTRATIGRÁFICO Que coincide con el final del período Formativo Ecuatoriano. El material cultural encontrado bajo ESTA TEFRA MUESTRA EVIDENCIA DE RELACIONES SOCIALES Y DE INTERCAMBIO ENTRE VARIOS GRUPOS HUMANOS CONTEMPORÁNEOS. ESTA INTERRELACión SE diO A NIVEL REGIONAL E INTERREGIONAL, EVIDENCIA DE ESTO ES LA PRESENCIA DE OBSIDIANA Y CERÁMICA DE CARACTERÍSTICAS COTOCOLLAO A LO LARGO DE LA SIERRA Y NOROCCIDENTE de Pichincha, así como cerámica con características Chorrera hacia el norte, sur y este en la costa, hasta AlCANZAR la SIERRa. DE ESTA RED DE RELACIONES SURGEN VARIAS CARACTERísticas SOCIOCULTURALES QUE Sugieren existía complejidad social al final del Período Formativo Tardío.

Palabras Claves: Período Formativo Tardío - complejidad social - Pululahua.

Abstract

In SEVERal archaeological sites between VAlley of Quito and Manabí province, tephra of the last eruptive cycle of the Pululahua volcano (2500-2400 BP) have been identified in the stratigraphy. IN AREAS AFFECTED BY THIS NATURAL PHENOMENON, THIS LAYER OF ASH IS A CHRONO-STRATIGRAPHIC REFERENCE that coincides with the end of the Ecuadorian Formative Period. The cultural material found under THIS TEPHRA SHOWS EVIDENCE OF SOCIAL RELATIONS AND EXCHANGES BETWEEN VARIOUS HUMAN GROUPS. THESE RELATIONSHIPS OCCURRED ON A REGIONAL AND INTERREGIONAL LEVEL. COTOCOLLAO OBSIDIAN AND POTTERY WAS

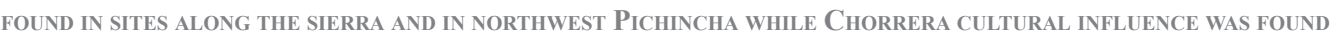
TO THE NORTH, SOUTH AND THE COAST TO THE EAST, INCLUDING THE HIGHLANDS. FroM THIS WEB OF RELATIONSHIPS, SEVERAL SOCIO-CULTURAL CHARACTERISTICS EMERGE THAT SUGGEST THERE WAS SOCIAL COMPLEXITY AT THE END OF THE LATE Formative PERIOD.

Keywords: Late Formative Period - social complexity - Pululahua.

* Este artículo es una síntesis de la disertación presentada para la obtención del título de Antropóloga con mención en Arqueología en la Universidad Católica del Ecuador.

* La autora ha colaborado en varios proyectos de investigación arqueológica, ha trabajado en el INPC y actualmente presta servicios profesionales en la Reserva Arqueológica Rumipamba del IMP (Instituto Metropolitano de Patrimonio). Correo electrónico: silvia figueroa@yahoo.com 


\section{Introducción}

T a erupción del volcán Pululahua de hace 2400 años $\mathrm{AP}^{1}$ afectó a varios asentamientos humanos que se encontraban en la zona del valle de Quito, su cordillera occidental, la planicie costera, hasta la isla de La Plata en la provincia de Manabí (Zeidler e Isaacson, 2003; Vallejo, 2011; Andrade et al., 2012). Bajo la tefra de este evento natural catastrófico quedó abundante material cultural relacionado con la ocupación y desarrollo de actividades domésticas, artesanales y agrícolas en estos sitios. El análisis minucioso del registro arqueológico, arrojó datos que evidencian la interacción de estos grupos humanos a nivel regional e interregional, al final del período Formativo Tardío.

En un análisis de cinco sitios (Cotocollao, Tajamar, Nueva Era, Nambillo y San Isidro) que fueron cubiertos por esta ceniza, la evidencia arqueológica sugiere que aquí existía una red de relaciones sociales y de intercambio que posiblemente ya estaba en marcha desde el período Formativo Temprano (Isaccson, 1987; Villalba, 1988; Zeidler, 1994; Lippi, 1998; Vásquez, 1999; Marcos, 2003; Valdez, 2008). La presencia de elementos culturales foráneos ${ }^{2}$ en estos sitios, permite inferir que sus formas de organización social posibilitaban el mantenimiento de esta dinámica de interacción con un flujo de información y bienes y sin un control centralizado. Este proceso de ingreso, procesamiento y salida de información nutre al sistema con nuevas ideas, tecnología, historias, experiencias, etc., que se traducen en cambios sociales y/o culturales.

Este modelo de interacción social puede entenderse a través de la Teoría de los Sistemas Complejos (Bentley y Maschner, 2008: 245; Mitchell, 2009: 13), donde para evaluar la complejidad de un sistema no se estudia sus componentes sino las propiedades que surgen de su interacción. Es decir, de la red de individuos y grupos humanos que estaban interactuando al final del Período Formativo Tardío, surgen unas propiedades sociales y culturales que le darán el carácter de complejidad social.

\section{Volcán Pululahua}

El complejo volcánico Pululahua (CVP), se encuentra a $20 \mathrm{~km}$. al norte de la ciudad de Quito y tiene una altura de 3356 m.s.n.m. en el Sincholahua, su domo más alto. El último período eruptivo de este volcán se inició hace 2500 años AP y duró aproximadamente cien años hasta el 2400 AP (Andrade et al., 2012: 27-28). La ceniza del volcán Pululahua es de un color blanquecino ${ }^{3} \mathrm{y}$ por eso es fácilmente identificable en la estratigrafía de los sitios que fueron afectados por la misma (Vallejo, 2011; Andrade et al., 2012).

La erupción más fuerte del Pululahua de hace 2400 años AP, cubrió con material volcánico una extensa zona entre la sierra y costa, ésta fue de tipo pliniano con una explosividad de nivel $5 \mathrm{VEI}^{4}$ (Vallejo, 2011: tabla 5.15, 146) y estuvo acompañada de varios eventos explosivos prolongados. Evidencia de esta ceniza se encontró hasta Cayambe al noreste, San Isidro (Manabí) al oeste, San Juan al sur de Quito entre los volcanes Ninahuilca y Atacazo y los alrededores de Mindo y Nanegalito por el Noroeste (Hall y Mothes, 1994: 29). Adicionalmente, en un trabajo más reciente se pudo analizar varias muestras recolectadas en sitios que van desde el sur de Esmeraldas hasta el norte de Santa Elena y se concluyó que en diez de éstas

1 Revisar Tabla 1: Recopilación de Dataciones para la última erupción del volcán Pululahua y para el final del Período Formativo Tardío en cinco sitios.

2 Artefactos, materia prima, influencia en la manufactura y aspectos decorativos de la cerámica, etc.

3 "Es fácilmente reconocible por su posición estratigráfica, por la naturaleza de su pómez blanca, de fragmentos líticos alterados de basalto y argilitas negras, y por su mineralogía característica." (Vallejo, 2011: 29)

4 V.E.I., Volcanic Explosivity Index, este índice es utilizado para describir la gravedad de una erupción explosiva y varía entre 0 (no explosivo), 1 (pequeña), 2 (moderada), 3 (moderada-grande), 4 (grande), 5 (muy grande) hasta 8. (Andrade et al., 2012: 103) 
existe evidencia de ceniza del Pululahua, estos sitios están entre San Isidro en el norte de Manabí y rio Juancho al sur de la misma provincia (Vallejo, 2011: 118-119). Este hecho, permite comparar sitios que fueron contemporáneos, es decir, esta tefra del Pululahua de hace 2400 años AP, es un marcador temporal que coincide con el final del período Formativo Tardío.

La situación sociocultural de las sociedades en el pasado durante estas etapas de crisis puede ser interpretada y entendida a partir de los datos que se obtienen en una excavación arqueológica y especialmente de lo que podemos leer en el registro estratigráfico. Por esta razón, el conocimiento del proceso eruptivo de un volcán permite interpretar o inferir el curso de ciertos procesos o cambios sociales como sucedió al final del período Formativo ecuatoriano.

\section{Sitios arqueológicos del período formativo tardío, afectados por la erupción del Pululahua de hace 2400 años AP}

El Período Formativo ecuatoriano es extenso (4000-500 a.C.), de aproximadamente 3500 años. Meggers Evans y Estrada (1965) establecieron las condiciones geográficas, medio ambientales y sociales para este período, solo considerando lo que habían observado en la costa: sedentarismo, desarrollo de la cerámica, agricultura, patrones de asentamiento y relaciones de intercambio (Marcos, 2003: 7-11). Estos procesos de desarrollo social y cultural fueron algo diferentes en la costa, sierra y oriente. Sin embargo, el registro arqueológico muestra que al final del período Formativo Tardío ecuatoriano, varios grupos humanos estaban en contacto a nivel regional e interregional. En cinco de estos sitios que fueron cubiertos por la ceniza del volcán Pululahua hace 2400 años AP, existe evidencia material de esta interacción.

Cotocollao (Villalba, 1988)

Cotocollao (1100 a.C. 500 a.C.) es un referente cultural para el Período Formativo ecuatoriano en la sierra, se encuentra en el sector del mismo nombre al norte de Quito. Según Villalba (1988), este sitio estuvo ocupado por alrededor de 1000 años y al parecer fue una aldea que basó su sustento en la cacería, recolección y agricultura. El sitio tuvo dos fases de ocupación una temprana que iría desde aprox. 1500 a 1100 a.C. seguida de una tardía que va desde 1100 a.C. hasta el 500 a.C. (Ibíd.: 245). Durante este tiempo se desarrolló la producción cerámica representada en 34 clases formales de vasijas, cuencos, ollas y botellas. Así mismo, el trabajo en lítica es muy representativo con la producción de cuencos de piedra, artefactos de obsidiana, basalto y andesita (Ibíd.: 271-309).

La población de Cotocollao se asentó principalmente en torno a un cementerio y otro grupo de viviendas bastante cercano sobre una colina (Villalba, 1988: 64-65). En la primera y segunda fase de Cotocollao se nota la insistencia por ocupar el espacio en torno al cementerio a pesar del aglutinamiento de las viviendas que al parecer ya existía. Posiblemente esto y los pocos elementos considerados como ajuar funerario en los enterramientos pueden considerarse como aspectos que marcan una diferenciación social por prestigio ${ }^{5}$ (Ibíd.: 71, 78-79, 101).

Cabe destacar, que Cotocollao tenía contacto con grupos humanos de la costa, del oriente y del callejón interandino, esto se observa en el material cultural foráneo encontrado en el sitio y de la cerámica Cotocollao en otros sitios a lo largo de la sierra principalmente (Arellano, 1992; 1994). Además, se debe mencionar que, en el cementerio tardío, se evidenció que 5 cráneos de los 27 analizados tenían deformación craneana (Ubelaker, 1980, en: Villalba, 1988: 566) similar a las observadas para esta misma etapa en la cultura Chorrera.

5 Recipientes cerámicos y de piedra, objetos de adorno corporal o utensilios de uso personal como orejeras, colgantes, cuentas de collar, etc. 


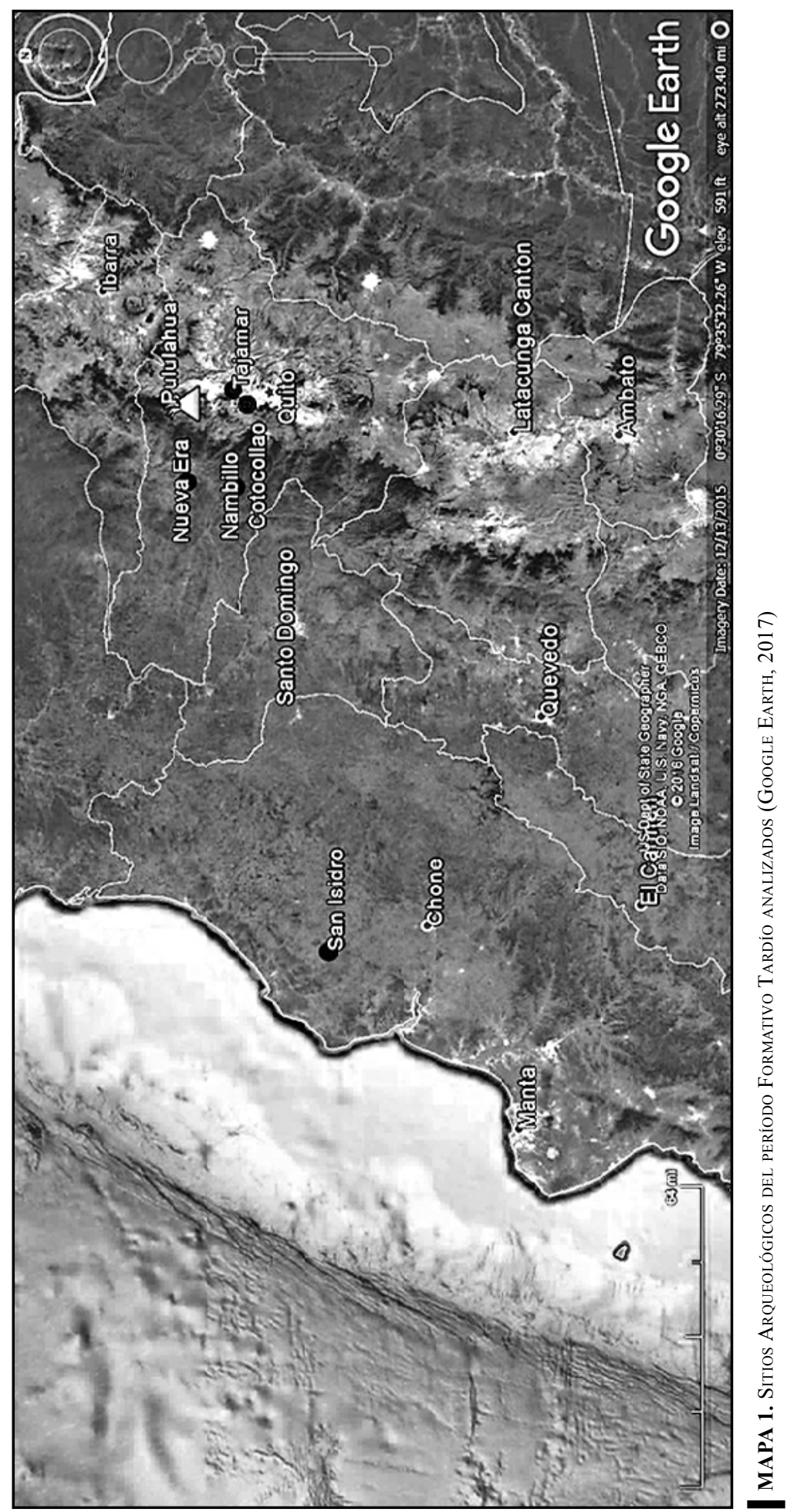


Tajamar (Domínguez, 2011)

Este sitio Formativo (1120-870 a.C. - 335 a.C.) también se encuentra en el área de Quito, en San Antonio de Pichincha. Aquí se distinguen dos momentos de ocupación, uno durante el Formativo tardío y otro durante el período de Integración. Del material cultural recuperado de este sitio y que corresponde al Formativo Tardío, se puede observar que el $80 \%$ de la cerámica guarda características similares a las de Cotocollao, pero con un patrón de asentamiento diferente, con viviendas circulares y enterramientos aislados de éstas y sin ajuar funerario (Domínguez, 2011: 22-25). Adicionalmente aquí se encontró abundante obsidiana y algunos restos de talla estaban asociados a fogones exteriores. (Ibíd.: 23). Cabe destacar que entre el material formativo de este sitio también se encontraron elementos foráneos, algunas conchas prietas ${ }^{6}$ trabajadas. (Ibíd.: 215-216).

\section{Nueva Era (Isaacson, 1987)}

Este sitio (1500-1200 a.C. - 335 a.C.) se encuentra en el Noroccidente de la provincia de Pichincha. Aquí se pudo identificar una ocupación que iría desde el Formativo Medio hasta el Formativo Tardío. El material cultural fue recuperado de un contexto doméstico, éste está bastante fragmentado y la mayoría tiene características similares a las de Cotocollao, aunque el acabado de algunos fragmentos es similar al observado en Chorrera (Isaacson, 1987: 217 $218,289)$. Así mismo, aquí se encontraron abundantes fragmentos de obsidiana y piedra verde como evidencia de la existencia de talleres de lítica (Ibíd.: 215). En este sitio existe evidencia de interacción con otros grupos humanos de la sierra y de la costa, visibles en los atributos decorativos y formas de varios fragmentos de cerámica. Al parecer el tipo de vivienda de Nueva Era tenía forma rectangular, por la disposición de los huecos de poste. En esta excavación no se encontraron tumbas.

Nambillo (Lippi, 1998)

Este sitio (1600 a.C. - 400 a.C.) también se encuentra hacia el occidente de Quito y se denominó Nambillo Temprano (Lippi, 1998a: 109-110). Aquí se identificó material cultural del Período Formativo Tardío como cerámica Cotocollao ${ }^{7}$ de carácter utilitario, lítica y abundante obsidiana (Ibíd.: 109, 244, 292). Según Lippi (1998b) la obsidiana posiblemente fue un objeto importante de intercambio ya que se ha encontrado evidencia de ésta en varios sitios de la costa y en esta zona del Pichincha occidental (Lippi, 1998b: 123-124).

San isidro (Zeidler y Pearsall, 1994)

Este sitio se encuentra a $25 \mathrm{~km}$. de la costa en la provincia de Manabí, con una historia de ocupación intermitente de aproximadamente 3000 años, desde Valdivia VIII hasta Jama Coaque II (Zeidler y Pearsall, 1994: 4). La ocupación Chorrera se da desde 1000 a.C. hasta la erupción del volcán Pululahua 335 a.C. (Ibíd.: Tabla 7.1, 113). Para este período se distinguen dos áreas diferenciadas de ocupación, una sobre la zona aluvial densamente poblada y la otra sobre un montículo artificial de forma cuadrangular. Este montículo fue inicialmente construido en Valdivia VIII y durante la ocupación Chorrera aumentó su tamaño, sin que este claro la función del mismo para uso ceremonial o habitacional (Ibíd.: 78-82). La influencia de la cerámica Chorrera se extiende por toda la costa (Ibíd.: 114) y hacia el este hasta alcanzar varios puntos en la región andina (Zeidler, 2003; 2008).

6 Molusco Bivalvo, familia Arcidae Lamarck, especie Anadara tuberculosa (Domínguez, 2009, Cap. 7: 1-4).

7 Cerámica Cotocollao de las clases formales II, VI, VII, XIV y XV. 


TABLA 1. Recopilación de Dataciones Para la Última erupción del volcán Pululahua y Para el
final del Período Formativo Tardío, en Cinco sitios
\begin{tabular}{|l|c|c|}
\hline Sitios & Formativo Tardío & Pululahua \\
\hline Cotocollao (Villalba, 1988) & 1100 a.C. - 500 a.C. & 2355 a A.P. \\
\hline Nueva Era (Isaacson, 1987) & $1500-1200$ a.C. -335 a.C. & 335 a.C. \\
\hline Nambillo Temprano (Lippi, 1998) & 1600 a.C. -400 a.C. & 2300 a A.P. \\
\hline San isidro (Zeidler y Pearsall, 1994) & 1000 a.C. -355 a.C. & 355 a.C. \\
\hline Tajamar (Domínguez, 2011) & $1120-870$ a.C. - 335 a.C. & 335 a.C. \\
\hline Marcos (2003) & 850 a.C.- 335 a.C. & 335 a.C. \\
\hline Andrade et al. (2012) & -------------------- & 2500 a 2400 a A.P. \\
\hline
\end{tabular}

Elaborado por la autora.

\section{Complejidad social}

Desde la arqueología tradicional, el estudio de la complejidad social ha destacado elementos socioculturales relacionados con el surgimiento de jerarquías, el acceso diferenciado a los recursos naturales, el prestigio, el aumento de la densidad poblacional, intensificación de la agricultura, etc. (Sahlins, 1958; Service, 1962, Mayers-Oakes, 1963; Fried, 1967; McGuire, 1983 y otros), como aspectos claves para el surgimiento de una diferenciación y/o estratificación social en los grupos humanos del pasado. Sin embargo, en estas sociedades existieron varios aspectos socioculturales que también dan cuenta de su complejidad social. Éstos surgen no solo del análisis de la organización social un grupo humano, sino de su relación con otros individuos y grupos humanos con los que formaron redes de interacción. Es decir que, si bien existen grupos humanos que tienen aspectos culturales y de organización social diferentes, la interacción con otros, el intercambio de bienes y principalmente de información, influye en el surgimiento de características socioculturales complejas (Dillehay, 2006; Bentley y Maschner, 2008; Mitchell, 2009, y otros).

En la organización social de los grupos humanos del pasado la diferenciación social pudo haber sido de tipo jerárquico, donde ciertos individuos o grupos humanos tenían acceso diferenciado a los recursos naturales (McGuire, 1983: 93). En cambio, si existe evidencia de una diferenciación social menos marcada donde prima el prestigio respecto de los roles sociales que ejerce algún miembro de la comunidad, la organización social es heterárquica (Ibíd.). Si bien en ambos casos existe una diferenciación social, la primera es de carácter vertical y establece fundamentalmente rangos o estratos dentro de la sociedad y la segunda es horizontal y la diferenciación es por estatus social.

Así mismo, los procesos de toma de decisiones también mostrarían aspectos relacionados con la complejidad social (Smith, 2011: 57). El agency como capacidad de acción colectiva, es un aspecto sociocultural donde se expresan los procesos cognitivos que resultan de la interacción con otros individuos y grupos humanos (Robb, 2005, 2010; Gardner, 2008). Si consideramos a los individuos y grupos humanos del pasado y del presente como elementos que interactúan y forman redes de interrelación, se puede entender que este contacto permanente estimula el intercambio principalmente de información. La información que se adquiere, se procesa y reinterpreta y puede plasmarse en el material cultural a través de rasgos tecnológicos y decorativos que cambian a través del tiempo y que son producto de esta interrelación con otros (Sillar, 2011). 
Las sociedades tienen aspectos socioculturales que son parte de su proceso de desarrollo y éstos son dinámicos. El intercambio de información alimenta este proceso y contribuye a que ciertos aspectos de su organización social no sean perennes. Esto es posible entenderlo a través de la teoría de sistemas complejos. Para Melanie Mitchell (2009: 13), un sistema complejo es " a system in which large networks of components with no central control and simple rules of operation give rise to complex collective behavior, sophisticated information processing,

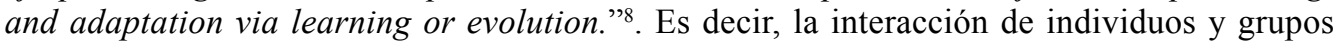
humanos del pasado formó una red de relaciones sociales y de intercambio de información y bienes, con un comportamiento colectivo complejo y sin un control centralizado. Un sistema así, al final de período Formativo Tardío hace 2400 años AP, se caracterizaría por tener aspectos socioculturales como:

- Diferenciación social (jerárquica o heterárquica)

- Especializaciones (talleres de lítica, cerámica, intercambio de bienes escasos, etc.)

- Interdependencia (intercambio de bienes e información)

- Capacidad de acción colectiva (procesos de toma de decisiones)

De esta forma, las propiedades emergentes de los sistemas complejos, son producto de esta interrelación entre sus componentes (Bentley y Maschner, 2008; Mitchell, 2009; Reilly y Linds, 2010). Entonces, el análisis de la complejidad social va a ser en torno a estos aspectos socioculturales que surgen de la interrelación de varios grupos humanos al final del período Formativo Tardío, más no del análisis individual de sus componentes.

\section{Evidencia de complejidad social al final del período formativo tardío}

Eventos naturales como las erupciones volcánicas de grandes dimensiones causan daño no solo en el entorno natural del volcán, sino que la ceniza expulsada hacia la atmósfera puede desplazarse a miles de kilómetros y afectar poblaciones alejadas. Estas erupciones pueden inclusive causar variabilidad en el clima, lo que ha contribuido a la afectación de cultivos y ciclos de cultivo y por ende provocar hambrunas y enfermedades. Los efectos inmediatos de estos eventos naturales pueden ir desde la pérdida de vidas humanas, contaminación de fuentes de agua, pérdida de cultivos, de flora silvestre y pérdida de otros recursos, todo lo cual puede provocar una migración forzada, con el abandono temporal o definitivo de un sitio, poniendo a prueba el nivel de organización social de un grupo humano y su capacidad de acción colectiva. Para quienes migran y pierden su espacio o lugar de pertenencia, su situación es de vulnerabilidad frente a factores de orden natural, social, político y religioso.

Hace 2500 años AP el volcán Pululahua inició su último ciclo eruptivo, éste duró aproximadamente cien años. Una erupción potente hace 2400 años AP cubrió con material volcánico extensas áreas entre el valle de Quito y la costa de la actual provincia de Manabí (Vallejo, 2011; Andrade et al., 2012). La erupción del volcán Pululahua hace 2400 años AP, provocó el abandono de varios sitios ocupados por grupos humanos de la sierra, ceja de montaña y costa. El material cultural analizado en los sitios Cotocollao, Tajamar, Nueva Era, Nambillo y San Isidro, estuvo cubierto por esta ceniza del Pululahua y muestra evidencia de que estas sociedades eran contemporáneas y de que estaban interactuando a nivel interregional desde mucho antes de esta erupción.

8 Un sistema en el que grandes redes de componentes sin un control central y con simples reglas de operación dan lugar a un comportamiento colectivo complejo, con un sofisticado procesamiento de la información y adaptación a través del aprendizaje o de la evolución. (traducido por la autora). 


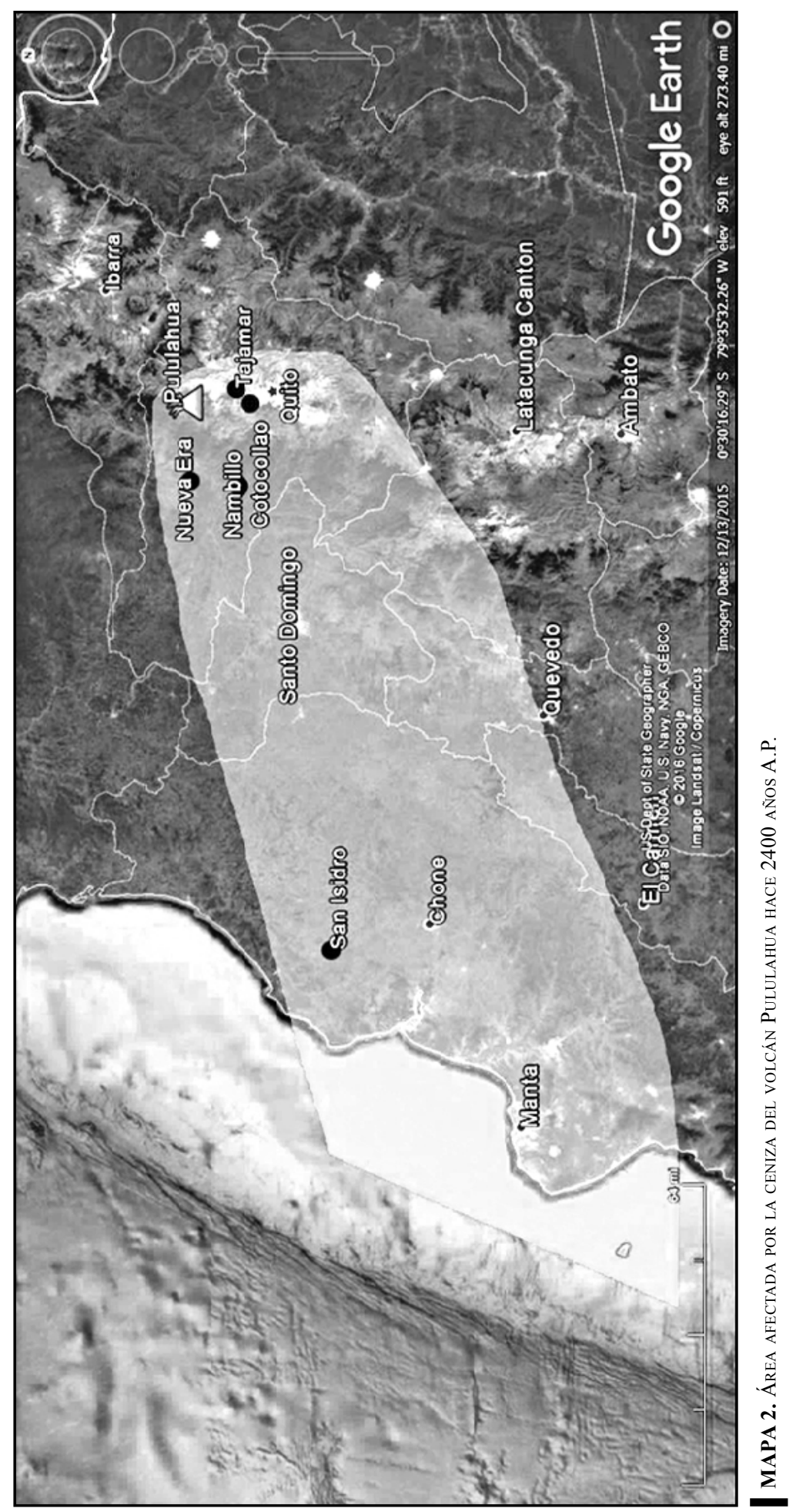


La influencia cultural que existió entre varios grupos humanos de este período y los cambios en la organización social, es posible observar en las técnicas de manufactura de la cerámica, atributos decorativos, artefactos elaborados con materias primas foráneas, artefactos foráneos, prácticas sociales, etc.

Como se mencionó anteriormente, la cultura Cotocollao es la más representativa para el período Formativo Tardío en la sierra y al parecer mantenía contacto con grupos humanos de la misma región, de la costa y oriente. Sin embargo, hay que considerar lo que Villalba (1988: 252) afirma, "Cotocollao resulta ser la síntesis más temprana y mejor documentada de una posible transición cerámica Valdivia-Machalilla". Es decir, la cerámica Cotocollao muestra algunos aspectos relacionados con la manufactura y decoración que son similares a los observados en la cerámica de culturas más tempranas como son Valdivia VIII y Machalilla.

La influencia de Cotocollao al final del Formativo Tardío se observa en otros sitios también cubiertos por la ceniza del Pululahua de hace 2400 años AP. Estos son Tajamar, Nueva Era y Nambillo con un alto porcentaje de su cerámica similar a la observada en Cotocollao. Además, Arellano (1992: 169; 1994: 118-120) afirma que existen sitios del Formativo Tardío a lo largo de la sierra desde el norte hasta la región austral donde se encuentra cerámica con formas y rasgos decorativos Cotocollao, al igual que la obsidiana de la región Quito.

Adicionalmente, tanto en la ceja de montaña del occidente, como en algunos sitios de la sierra también se ha encontrado cerámica donde se identifica la influencia de la cultura Chorrera9. Este es el caso de Nueva Era (Isaacson, 1987), Nambillo (Lippi, 1998), Las Mercedes y Los Naranjos (Constantine, 2014), Loma Pucará (Arellano, 1992, 1994), etc., confirmando que la interacción de grupos humanos a nivel interregional ya se daba desde mucho antes, posiblemente desde el período Formativo temprano.

En este sentido, el contacto con otros grupos humanos provocó un intercambio de saberes ${ }^{10}$ que se reflejan especialmente en aspectos morfofuncionales y atributos decorativos de la cerámica. Al ser la cerámica un indicador del grado de desarrollo tecnológico alcanzado por las sociedades del pasado, ésta permite rescatar aspectos sociales y culturales como la diferenciación social, ideología, especializaciones, así como la interacción con otros grupos de diferente tradición cultural (Ghezzi, 2011: 2).

Con esta evidencia, se puede decir que, al final del período Formativo Tardío se tiene a varios grupos humanos que están interactuando y forman una red de relaciones sociales y de intercambio dentro de una dinámica en la que no existe un control centralizado a pesar de que sí existen formas de diferenciación social por prestigio y/o por rango ${ }^{11}$. En este contexto, un evento catastrófico como una erupción volcánica podría acelerar estos procesos de cambio debido a la situación de vulnerabilidad de una parte o de toda la población. Entonces, aquí se pone en evidencia el nivel de organización social de una población, su capacidad de acción colectiva, su infraestructura, nivel tecnológico, conocimiento de su medioambiente, relaciones sociales y de intercambio, creencias religiosas, grado de interdependencia de objetos foráneos etc., es decir, aspectos relacionados con lo social y cultural que podrían modificar las características de este sistema.

9 Acabado de superficie (cerámica pulida con guijarro), fragmentos de pico de botellas silbato y asa de estribo.

10 Información que ingresa, se procesa, reinterpreta y sale.

11 En la zona central de la costa existe evidencia de que para el final del período Formativo Tardío estaba en marcha un proceso de cambio social con indicios de jerarquización. 


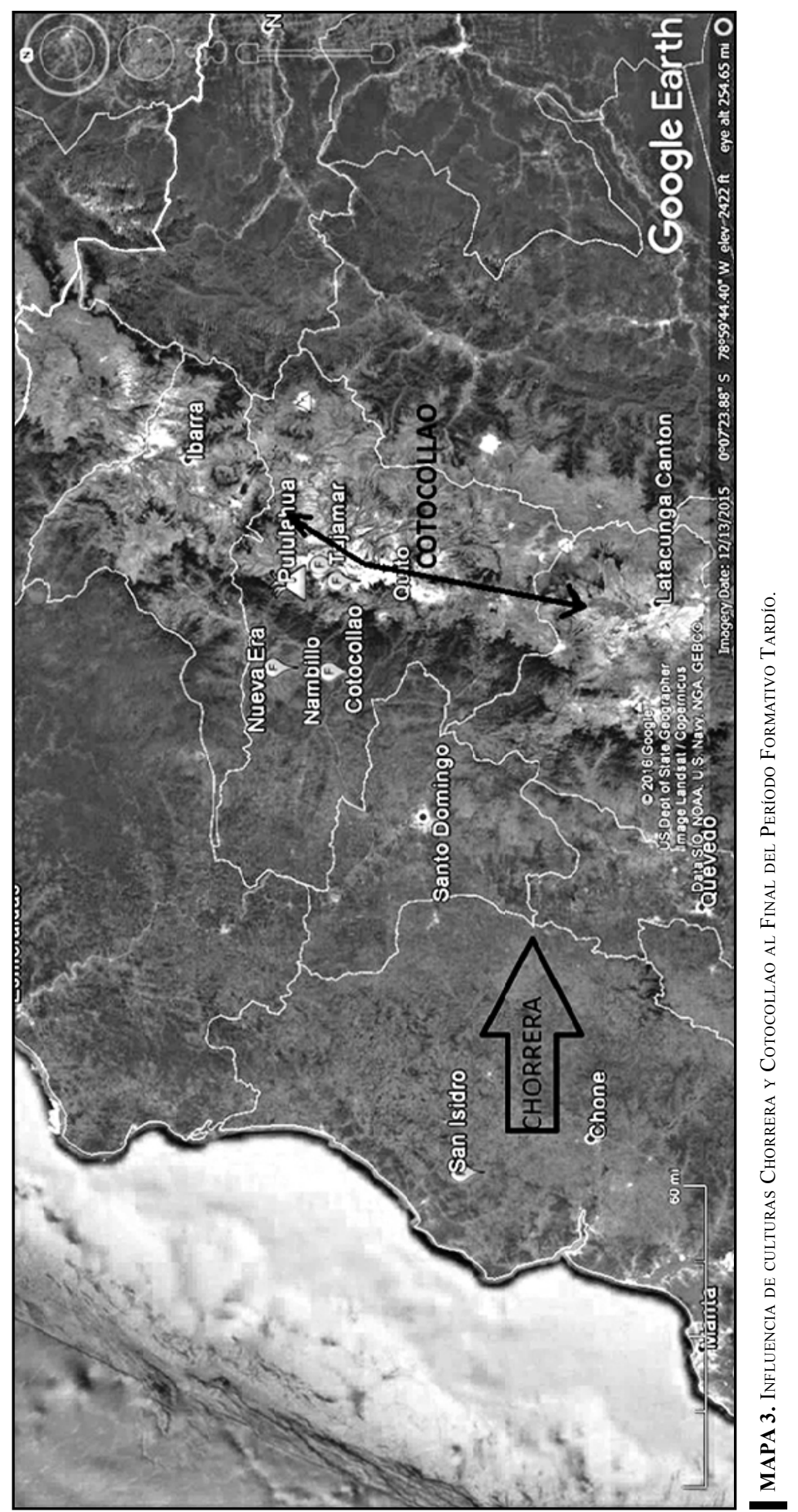




\section{Conclusiones}

Las sociedades del pasado y presente, no son entes estáticos, éstas pasan por procesos sociales dinámicos y están siendo permanentemente realimentados por la información y conocimiento alcanzados. Por esta razón, el cambio social, adaptarse y evolucionar, es un proceso normal de las sociedades (Mitchell, 2009).

Los aspectos sociales y culturales que surgen de la interacción entre individuos y grupos humanos del período Formativo Tardío a través de sus relaciones sociales y de intercambio, son evidencia de complejidad social.

La erupción del Pululahua provocó una migración forzada con el abandono temporal o definitivo de varios sitios de ocupación. En áreas no afectadas debe haber aumentado la densidad poblacional. Sin embargo, quienes migran se enfrentan a una situación de vulnerabilidad por la desigualdad social, cultural, política, lo que puede también motivar una diferenciación social jerárquica. Esta erupción de hace 2400 años AP coincide con el fin del período Formativo ecuatoriano. Esto es visible en el registro arqueológico donde se observa un cambio sociocultural que se expresa en la paulatina desaparición de las tradiciones cerámicas Chorrera (costa) y Cotocollao (sierra) y el surgimiento de nuevas expresiones culturales que marcan el período del Desarrollo Regional. Las nuevas formas de organización social dieron lugar a la formación de grupos humanos con marcados rasgos étnicos, con un aumento en su densidad poblacional, incremento de la producción agrícola y de bienes y una intensa relación interregional. 


\section{Bibliografía}

Andrade, D. et al. 2012, Los peligros volcánicos asociados con los volcanes Atacazo-Ninahuilca y Pululahua, Instituto Geofísico Escuela Politécnica Nacional e IRD Institut de Recherche pour le Développement, Corporación Editora Nacional, Quito.

Arellano J. 1994, “Loma Pucara, a Formative site in Cebadas Valley, Ecuador”, en: National Geographic research and exploration, $\mathrm{N}^{\circ} 10(1)$, pp. 118-120.

1992, "Primeras evidencias del Formativo tardío en la Sierra central del Ecuador", en: Ledergerber, P. (Ed.) Formativo sudamericano, una revaluación, Abya-Yala, Quito, pp.163-178.

Bentley, A. y Maschner H. 2008, “Complexity Theory”, en: Bentley A. y H. Maschner (Eds.) Handbook of Archaeological Theories, Altamira Press, Kindle Edition, 15, pp. 245-270.

Constantine, A. 2014, "La tecnología lítica de los cazadores recolectores tempranos del piedemonte andino occidental: sitios Las Mercedes y Los Naranjos, Provincia Santo Domingo de Los Tsáchilas, Cantón Alluriquín-Parroquia Las Mercedes", Tesis de Posgrado previa a la obtención del Título de Magíster en Arqueología del Neotrópico, en la Facultad de Ingeniería en Ciencias de la Tierra, Corporación Nacional de Arqueología, Antropología e Historia de la ESPOL, Guayaquil.

Dillehay, T. 2006, “Organización y espacios sociopúblicos incipientes: tres casos de los Andes”, en: Boletín de Arqueología PUCP, 13, pp. 13-36.

Dobres, M. y J. Robb. 2000, “Agency in Archaeology, Paradigm or platitude”, en: Dobres, M. y Robb, J. Agency in Archaeology, Routledge, New York, 1, pp. 3-17.

Domínguez, V. 2009, Prospección intra-sitio, excavación y monitoreo en el área de Ciudad Bicentenario-sitio arqueológico Tajamar Z3B1-017 (lado sur)-primera parte, Informe (inédito), INPC.

Figueroa, S. 2015, "Evidencia de Complejidad Social al final del período Formativo Tardío, bajo la ceniza del volcán Pululahua (2400 a A.P.)", Disertación previa a la obtención del título de Antropóloga con mención en Arqueología. Facultad de Ciencias Humanas Pontificia Universidad Católica del Ecuador, Quito.

Gardner, A. 2011, "Action and Structure in Interpretative Archaeologies", en: Cochrane, E. y A. Gardner (Eds.) Evolutionary and Interpretative Archaeologies, Left Coast Press, Inc. Walnut Creek, 3, pp. 63-82.

2008, “Agency”, en: Bentley, A. y Maschner, H. (Eds.) Handbook of Archaeological Theories, Altamira Press, Kindle Edition.

Gutiérrez, A. 2009, Dioses, Símbolos y Alimentación en los Andes, Interrelación hombre-fauna en el Ecuador Prehispánico, Abya-Yala, Quito.

Hall, M. y Mothes, P. 1998, "La actividad volcánica del holoceno en el Ecuador y Colombia austral, impedimento al desarrollo de las civilizaciones pasadas", en: Mothes, P. (Comp.) Actividad Volcánica y pueblos precolombinos en el Ecuador, Abya-Yala, Quito, pp.11-40.

Isaacson, J. 1987, "Volcanic activity and human occupation of the Northern Andes: The application of tephrostratigraphic techniques to the problem of human settlement in the western montana during the Ecuadorian formative", Tesis presentada en cumplimiento parcial de los requisitos para el grado de Doctor en Antropología en la Facultad de Postgrado de la Universidad de Illinois, Urbana.

Isaacson, J.y Zeidler J. 1998, "Accidental History: Volcanic activity and the end of the Formative in Northwestern Ecuador”, en: Mothes, P. (Comp.) Actividad Volcánica y Pueblos Precolombinos en el Ecuador, Abya-Yala, Quito pp. 41-72.

Lippi, R. 2013, Proyecto Arqueológico Palmitopamba, Informe Sobre la Temporada de Campo de 2012, INPC.

2003, "Formative Period Chronology for the Northern and Central Highlands of Ecuador", en: Raymond, S. y R. Burger. (Eds.) Archaeology of Formative Ecuador, Apéndice B. Dumbarton Oaks, Washington, pp. 529-537.

1998b, "Encuentros Precolombinos entre serranos y costeños en el país yumbo (Pichincha occidental, Ecuador)", en: Cárdenas, F. y T. Bray (Eds.) Intercambio y Comercio entre Costa, Andes y Selva. Arqueología y etnohistoria de Suramérica, Departamento de Antropología, Universidad de los Andes, Bogotá, pp. 115-134.

1998a, Una Exploración Arqueológica del Pichincha Occidental, Ecuador, Museo Jijón y Caamaño-PUCE, Quito.

1988, "Paleotopography and Phosphate Analysis of a Buried Jungle Site in Ecuador", en: Journal of Field Archaeology, 15 , pp. 85-97.

Marcos, J. 2003, “A Reassessment of the Ecuadorian Formative”, en: Raymond, S. y R. Burger (Eds.) Archaeology of Formative Ecuador, Dumbarton Oaks, Washington, pp. 1-27.

Mcguire, R. 1983, "Breaking down Cultural Complexity: Inequality and Heterogeneity, en: Advances in Archaeological Method and Theory, 6, pp. 91-142.

Mitchell, M. 2009, Complexity, a guide tour, Oxford University Press, New York.

Mothes, P. (Comp.) 1998, Actividad Volcánica y los Pueblos Precolombinos del Ecuador, Abya-Yala, Quito.

Raymond, S. y Burger, R. (Eds.) 2003, Archaeology of Formative Ecuador, Dumbarton Oaks, Washington. 
Reilly, R. y W., Linds. 2010, “Complexity”, en: Mills, A. et al. (Eds.) Encyclopedia of Case Study Research. Vol.1. SAGE Publications, Inc., Londres, pp. 180-182.

Robb, J. 2010, "Beyond Agency", World Archaeology. 42(4), pp. 493-520.

2005, "Agency", en: Renfrew, C. y P., Bahn (Eds.) Archaeology the key concepts, Routledge. New York, pp. 2-5.

Sillar, B. 2011, "Intentionality Matters: Creativity and Human Agency in the construction of the Inca State", en: Cochrane, E. y A., Gardner (Eds.) Evolutionary and Interpretative Archaeologies, Left Coast Press, Inc. Walnut Creek, 5, pp.105-125.

Smith, M. 2011, "What it takes to get complex: food, goods, and work as shared cultural ideals from the beginning of sedentism", en: Smith, M. (Ed.) The Comparative Archaeology of Complex Societies, Cap. IV, Cambridge University Press, Cambridge, pp. 44-61.

Ugalde, M. 2012, Rescate arqueológico de cementerio prehistórico en el barrio El Condado de la ciudad de Quito, Informe (inédito), Ministerio Coordinador de Patrimonio Cultural, Quito.

2013a, Proyecto de Prospección arqueológica Rancho Bajo, Provincia de Pichincha, Informe Final, Instituto Metropolitano de Patrimonio, Quito.

2013b, Informe Contrato Complementario del Proyecto de Prospección arqueológica Rancho Bajo, Provincia de Pichincha, Instituto Metropolitano de Patrimonio, Quito.

Valdez, F. 2008, "Inter-zonal Relationships in Ecuador", en: Silverman, H. y W. Isbell (Eds.) Handbook of South American Archaeology, Springer, Nueva York, pp. 865-888.

Vallejo, S. 2011, "Distribución de las Cenizas Holocénicas-Tardías en la Costa del Ecuador", Tesis de grado previa a la obtención del título de Ingeniera Geóloga de la Escuela Politécnica Nacional, Quito.

Villalba, M. 1988, Cotocollao: una aldea Formativa del Valle de Quito, Museos del Banco Central del Ecuador, Quito.

Villalba, M. y Alvarado, A. 1998, "La arqueología del Valle de Quito en clave volcánica", en: Mothes, P. (Coord.) Actividad Volcánica y Pueblos Precolombinos en el Ecuador, Abya-Yala, Quito, pp. 73-110.

Zeidler, J. 2003, "Formative period Chronology for the coast and western low lands of Ecuador", en: Raymond, S. y Burger, R. (Eds.) Archaeology of Formative Ecuador. Dumbarton Oaks, Appendix A, Washington, pp. 487-527.

2008, "The Ecuadorian Formative", en: Silverman, H. y W. Isbell (Eds.) Handbook of South American Archaeology, Springer, Nueva York, 24, pp. 459-488.

Zeidler, J. y Isaacson, J. 2003, "Settlement Process and Historical Contingency in the Western Ecuadorian Formative", en: Raymond, S. y Burger, R. (Eds.) Archaeology of Formative Ecuador, Dumbarton Oaks. Washington, pp. 69-123.

Zeidler, J. y Pearsall, D. (Eds.) 1994, Arqueología Regional del Norte de Manabí, Ecuador: Medio Ambiente, Cronología Cultural y Subsistencia Prehistórica en el Valle del Río Jama, Vol. 1, University of Pittsburgh, Department of Anthropology y Ediciones Libri Mundi, Quito. 